\title{
Nível de conhecimento e medidas de prevenção de moradores sobre a Leishmaniose Visceral em área endêmica no Maranhão, Brasil
}

\author{
Level of knowledge on Visceral Leishmaniasis and prevention measures of residents in an endemic area \\ of Maranhão, Brazil
}

Nivel de conocimiento y medidas de prevención de moradores sobre la Leishmaniasis Visceral en área endémica en Maranhão, Brasil

\author{
Gabriela de Sousa LOPES ${ }^{1}$ \\ Vilson de Almeida SOUSA ${ }^{2}$ \\ Jesuino da Silva Costa MARTINS ${ }^{2}$ \\ Elson Silva de SOUSA ${ }^{2}$ \\ Reinaldo Lucas CAJAÍBA2
}

${ }^{1}$ Graduanda do curso de Licenciatura em Biologia, Instituto Federal do Maranhão, campus Buriticupu. IFMA. 65393000 Buriticupu-MA, Brasil ${ }^{2}$ Laboratório de Ecologia e Conservação, Instituto Federal do Maranhão, campus Buriticupu. IFMA. 65393000 Buriticupu-MA, Brasil

\begin{abstract}
Resumo
Introdução: A Leishmaniose Visceral é uma enfermidade com ampla distribuição pelo Brasil e pode afetar tanto animais como humanos. As diversas ações antrópicas vêm contribuindo para sua disseminação, o que configura a temática como sendo de grande relevância para a saúde pública. Objetivos: O presente estudo foi desenvolvido em uma área endêmica no estado do Maranhão com o objetivo de avaliar o nível de conhecimento da população entrevistada sobre a Leishmaniose Visceral e conhecer quais são as medidas profiláticas adotadas. Material e Método: Foram aplicados questionários a 106 moradores da área urbana com idade variando entre 18 a 76 anos. Resultados: Os resultados demonstraram que o nível de conhecimento da população sobre essa zoonose é precário, pois a maioria desconhece os sintomas e formas de transmissão da doença. Conclusão: Fica evidente a necessidade do desenvolvimento de ações públicas educativas para prevenção e controle da leishmaniose, tal como a divulgação de informações por meio de palestras, por exemplo, para reduzir os índices de notificação no município e elevar o conhecimento da população.
\end{abstract}

Descritores: Conhecimentos, Atitudes e Prática em Saúde; Prevenção de Doenças; Leishmaniose Visceral.

\begin{abstract}
Introduction: Visceral leishmaniasis is a disease with widespread distribution in Brazil, affecting both animals and humans. The actions developed by the man have contributed to its dissemination, being thus of great relevance in public health. Objectives: The present study was carried out in an endemic area in the State of Maranhão, Brazil, with the objective of assessing the level of knowledge of the population on Visceral Leishmaniasis and what are the prophylactic measures adopted. Material and Method: We applied questionnaires to 106 residents of the urban area, ranging in age from 18 to 76 years. Results: The results showed that the level of knowledge of the population about this zoonosis is precarious, since most are unaware of the symptoms and forms of transmission of the disease. Conclusion: It is evident the need to develop actions aimed at prevention and control of leishmaniosis, as well as the dissemination of information through lectures, for example, to reduce the notification rates in the municipality and increase knowledge of the population.
\end{abstract}

Descriptors: Health Knowledge, Attitudes, Practice; Disease Prevention; Leishmaniasis, Visceral.

\section{Resumen}

Introducción: La Leishmaniasis Visceral es una enfermedad con amplia distribución en Brasil, pudiendo afectar tanto animales y humanos. Las acciones desarrolladas por el hombre vienen contribuyendo para su diseminación, siendo así de gran relevancia en la salud pública. Objetivos: El presente estudio fue desarrollado en un área endémica en el estado de Maranhão con el objetivo de evaluar el nivel de conocimiento de la población sobre la Leishmaniasis Visceral y cuáles son las medidas profilácticas adoptadas por los moradores. Material y Métodos: Se aplicaron cuestionarios a 106 habitantes del área urbana con edad variando entre 18 a 76 años. Resultados: Los resultados obtenidos demostraron que el nivel de conocimiento de la población sobre esta zoonosis es precario, pues la mayoría desconoce los síntomas y formas de transmisión de la enfermedad. Conclusión: Es evidente la necesidad del desarrollo de acciones dirigidas a la prevención y control de la leishmaniasis, así como a la divulgación de informaciones a través de charlas, para reducir los índices de notificación en el municipio y elevar el conocimiento de la población.

Descriptores: Conocimientos, Actitudes y Práctica en Salud; Práctica en Salud; Prevención de Enfermedades; Leishmaniasis Visceral.

\section{INTRODUÇÃO}

A Leishmaniose é uma enfermidade que resiste por longos estágios e apresenta uma variedade de síndromes complexas. Acomete hospedeiros animais e humanos. Sua transmissão ocorre principalmente através da picada de várias espécies fêmeas do inseto do gênero Lutzomya infectado pelo protozoário do gênero Leishmania ${ }^{1-2}$.

Apresenta-se de duas formas: a Leishmaniose Cutânea (LC), identificada por lesões na pele e nas mucosas das vias superiores e a Leishmaniose Visceral (LV), forma mais grave, sendo a febre alta um dos sintomas mais evidentes. Afeta órgãos como baço, fígado e intestino, além do quadro de anemia e sinais de desnutrição ${ }^{3}$. Caso não haja tratamento adequado, a doença pode ocasionar a morte do hospedeiro ${ }^{4}$.
No Brasil, as espécies de protozoários mais comumente identificadas são a Leishmania chagasi que causa LV tanto em humanos quanto em cães ${ }^{5}$, sendo a de maior preocupação no país ${ }^{6}$ e a Leishmania donovani, responsável pela infecção somente em humanos ${ }^{5}$. O vetor da leishmaniose é conhecido como mosquito-palha, birigui, asa branca ou tatuquiras conforme localidade . $^{7}$.

A LV está entre as doenças negligenciadas com elevado índice de distribuição no Brasil $^{8}$ que vem se ampliando com a decorrência do processo de urbanização ${ }^{9}$, aumento da população, fluxo migratório, desflorestamento em espaços para estabelecer novos povoados, progresso e a expansão da agricultura e atividades de extrativismo ${ }^{10}$. Sendo um decorrente problema de bem-estar social ${ }^{11}$. 
A compreensão das pessoas sobre o agente transmissor, sintomas e medidas profiláticas conduz a subsídios para a moderação da doença ${ }^{12}$. Vale ressaltar a importância do avanço nos estudos sobre a doença, principalmente nos municípios do estado do Maranhão que sofre de falta de saneamento básico adequado, fluxo migratório e constante ação antrópica em relação ao desmatamento e queimadas ${ }^{9}$. Avaliar o conhecimento das populações locais sobre a doença é de fundamental importância, pois é a partir deste entendimento que as medidas preventivas e atitudes podem ser executadas com maior eficiência e contribuir para a eliminação ${ }^{13}$.

Nessa perspectiva, este trabalho teve o objetivo avaliar o nível de conhecimento de moradores do município de Buriticupu-MA sobre a LV e conhecer quais são as medidas de prevenção adotadas, identificando e relacionando fatores ambientais e demográficos que possam influenciar em sua expansão.

\section{MATERIAL E MÉTODO}

\section{- Descrição da área de estudo}

$\mathrm{O}$ estudo foi desenvolvido na zona urbana do município de Buriticupu, Maranhão, localizado na Pré-Amazônia maranhense, a aproximadamente 430 $\mathrm{km}$ da capital São Luís (Figura 1). A extensão territorial é de 2.545,44 $\mathrm{km}^{2}$ e uma população estimada em 71.979 habitantes $^{14}$. O relevo é constituído por formações de tabuleiros, separados em faixas por drenos e grotões, situado a uma altitude de $200 \mathrm{~m}$ acima do nível do $\mathrm{mar}^{15}$. Encontra-se inserido em sua totalidade no ambiente geológico conhecido como Bacia Sedimentar do Pindaré, que atinge 12,40\% das Bacias do Estado do Maranhão. As principais atividades econômicas são a produção extrativa vegetal, pecuária e fruticultura ${ }^{14}$. O município de Buriticupu é o de maior prevalecimento da doença no estado do Maranhão e os conhecimentos da população são limitados, principalmente em relação ao tratamento ${ }^{16}$.

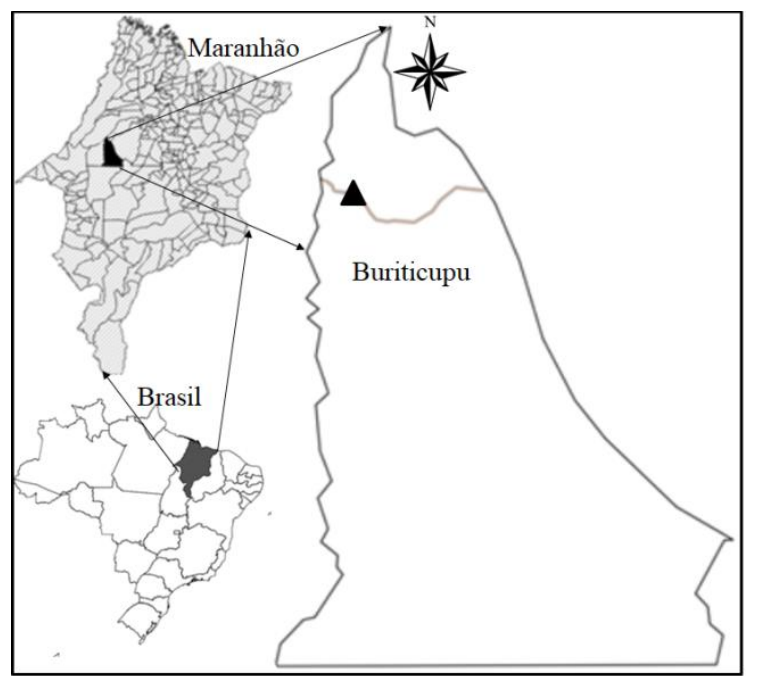

Figura 1: Localização do município de Buriticupu, Maranhão. O triângulo representa o perímetro urbano (adaptado de Lima et al. ${ }^{15}$ ).

\section{- Instrumento de coleta dos dados}

Para o estudo sobre o nível de conhecimento da população em relação a $L V$, foram selecionados os dois bairros da área urbana com maior registro de ocorrência da LV, com base nos dados da Secretaria Municipal de Saúde.

A pesquisa foi executada por intermédio de questionário semiestruturado contendo 16 questões abertas e fechadas, no período de janeiro a dezembro de 2017. As questões abordaram variáveis sociais e de conhecimentos dos entrevistados sobre características epidemiológicas, preventivas e ações de educação sobre a LV.

A autorização de participação foi obtida pela leitura e assinatura do Termo de Consentimento Livre e Esclarecido (TCLE), a fim de assegurar o anonimato dos sujeitos da investigação, as entrevistas foram numeradas de acordo com o prosseguimento em que se sucedeu. Foram entrevistadas apenas pessoas com idade igual ou superior a 18 anos.

\section{RESULTADOS}

Foram entrevistadas 106 pessoas, com idade entre 18 e 76 anos (média 41,70, dp $\pm 16,22$ ), sendo a maioria pertencente ao sexo feminino (59\%). O tempo de estudo dos entrevistados variou de zero a quinze anos e 18\% nunca estudou (analfabetos). Do total dos entrevistados, $58 \%$ afirmaram já ter ouvido falar sobre a LV e 76\% informaram que os Agentes Comunitários de Saúde (ACS) nunca discursaram sobre a temática. Ainda, 95\% informaram que nunca assistiram palestras sobre a temática, nem em escolas, hospitais ou qualquer outro meio de divulgação.

Quando questionados sobre a forma de obtenção de conhecimento sobre a LV, $21 \%$ citaram as conversas informais entre os vizinhos, $11 \%$ relataram ter ouvido principalmente em hospitais/unidades de saúde ou em situações em que a doença acometeu algum familiar/ amigo/ vizinho (Gráfico 1).

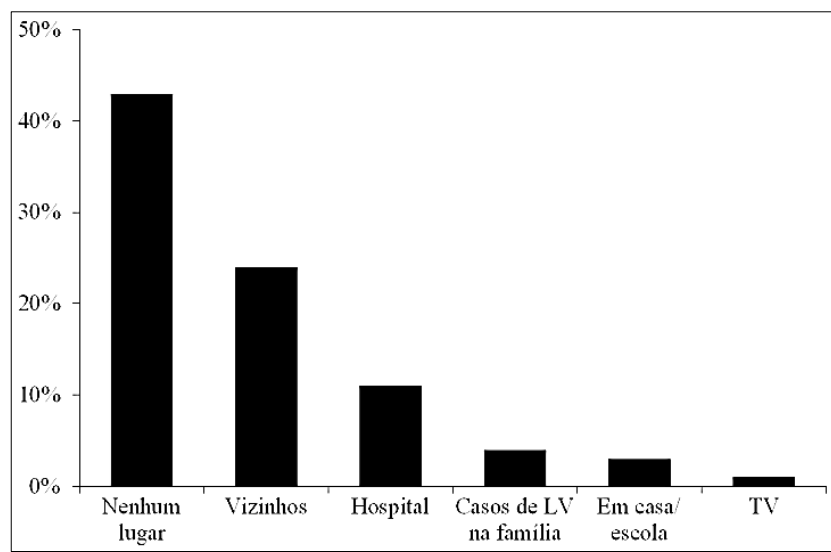

Gráfico 1: Principais formas de obtenção de conhecimento sobre a LV de acordo com os entrevistados.

No tocante ao conhecimento dos entrevistados sobre $\mathrm{o}$ agente transmissor da parasitose, 53\% não souberam opinar sobre quem 
transmite a doença, 28\% apontam o cachorro como o principal transmissor da LV, $10 \%$ o mosquito e $4 \%$ indicaram mosquito e cachorro, $3 \%$ cachorro e gato. Apenas $1 \%$ reconheceu o mosquito fêmea como o responsável.

Em relação à forma de contração da doença, $81 \%$ dos entrevistados desconhecem como se contrai a LV e $19 \%$ indicaram que sabem como adquiri-la. Dentre as manifestações clínicas citadas pelos colaboradores identificou-se que $76 \%$ desconhecem os sintomas, enquanto apenas $7 \%$ responderam corretamente (Tabela 1).

Tabela 1. Principais manifestações clínicas da LV de acordo com os entrevistados.

\begin{tabular}{lc}
\hline \multicolumn{1}{c}{ SINTOMAS } & $\mathbf{\%}$ \\
\hline Não sabe & $76 \%$ \\
Manchas no corpo & $3 \%$ \\
Febre e frio & $3 \%$ \\
Coceira & $3 \%$ \\
Febre e anemia & $2 \%$ \\
Ferimento na pele & $2 \%$ \\
Emagrecimento & $2 \%$ \\
Febre e inchaço & $2 \%$ \\
Dores de cabeça, febre e fraqueza & $2 \%$ \\
Dor e inchaço no corpo & $1 \%$ \\
Cãibras de sangue & $1 \%$ \\
Febre, vermelhidão no corpo, caroços e coceira & $1 \%$ \\
Perda de peso, amarelidão e febre & $1 \%$ \\
Dores abdominais & $1 \%$ \\
Barriga inchada, febre e cãibras de sangue & $1 \%$ \\
\hline Fonte: Dados da Pesquisa &
\end{tabular}

Quanto ao conhecimento sobre a cura da LV, $60 \%$ dos entrevistados afirmam que há cura, 23\% informaram que não há cura, enquanto $17 \%$ não souberam informar. Os entrevistados foram questionados sobre quais medidas preventivas eram adotadas contra a LV: $61 \%$ da população amostrada não utilizam nenhum tipo de medida; $37 \%$ utilizavam algum tipo de repelente químico para combater a LV (pesticidas de ação rápida a base de Praletrina, Cipermetrina, Imiprotrina, Solventes, Propelentes e Mascarante, por exemplo).

Questionados sobre a época de maior ocorrência da LV, 67\% informaram que não sabem a estação de maior ocorrência; $25 \%$ apontam o período chuvoso; $6 \%$ afirmaram que a ocorrência está propícia em todo ano; $3 \%$ indicam o verão como época de maior incidência.

\section{DISCUSSÃO}

Os resultados apresentados neste trabalho são corroborados com outros estudos realizados em várias regiões do nordeste ${ }^{16,17}$, onde se verifica que, em geral, há um pequeno nível de instrução e um elevado percentual no qual o ensino não foi satisfatoriamente desenvolvido ${ }^{18}$.

A falta de informações com relação à doença é um fator preocupante, além da falta de dados sobre a enfermidade no Brasil. Estudos têm demonstrado uma relação direta entre o índice de contágio da LV com baixa escolaridade ${ }^{13-19}$. Essa comparação pode ser explícita pela maior parte dos analfabetos viverem entre indivíduos de baixo poder aquisitivo, que também é a que mais sofre com a doença ${ }^{13}$. Por outro lado, a escolarização desempenha grande influência em peculiaridades como a obtenção de conhecimento e progresso de saúde dos cidadãos ${ }^{20}$.

É indispensável o desenvolvimento de projetos e capacitações junto ao poder público e o estabelecimento da real importância de discussões que essas endemias devem ter na saúde pública ${ }^{20}$. Dessa forma, trabalhos informativos que façam uso dos recursos de informação são imprescindíveis para a compreensão da doença.

Os ACS têm como uma de suas atribuições à divulgação de informações de casa em casa, assim, a capacitação desses profissionais deve ser constante ${ }^{21}$, pois a divulgação de informações efetuadas pelos ACS apresenta efetividade limitada, se estes não obtiverem um conhecimento preexistente sobre as doenças parasitárias ${ }^{22}$.

Em pesquisa realizada sobre o nível de conhecimento de moradores no município de Buriticupu Maranhão sobre Leishmaniose tegumentar ${ }^{16}$, verificou-se que $72 \%$ dos indivíduos detém baixo conhecimento em relação aos meios de transmissão da LV, dessa forma, necessita-se, portanto, de maiores informações quanto ao reservatório da doença bem como sua forma de transmissão. Informação esta que pode ser considerado como fator de prevenção reduzindo os riscos de ocorrência independentemente de sua localidade ${ }^{13}$. Até mesmo em regiões com elevado grau da enfermidade, a falta de discernimento em relação à transmissão, tratamento e precaução da doença são amplos, o que dificulta na implantação de medidas de contenção ${ }^{23}$. Portanto, o desenvolvimento de atividades e palestras de educação em saúde, bem como a utilização de diferentes meios como panfletos informativos são recursos que constituem o conhecimento e beneficiam a população ${ }^{24}$.

Além do homem, há outros tipos de reservatórios, como os mamíferos, por exemplo, os cães, roedores, marsupiais $\mathrm{e}$ as raposas. A proximidade de animais com as residências demonstra ser um fator de risco para a infecção da Leishmania ${ }^{9}$.

Os sintomas atribuídos incorretamente pelos moradores a LV, revelados na figura 1 demonstra que ações de educação e saúde devem ser efetuadas com urgência. A falta de informações sobre o conjunto de sintomas causados pela LV aponta a carência em práticas educativas ${ }^{13}$.

As instituições de ensino, assim como os veículos de comunicação, sejam eles televisão ou rádio, por exemplo, são meios importantes na divulgação de informações, desde as formas de transmissão e precaução, objetivando a redução de 
acontecimentos na cidade ${ }^{25}$.

Há uma grande chance de o indivíduo obter a cura da LV, desde que seja realizado o diagnóstico precoce da doença, seguido do tratamento correto ${ }^{26}$, do contrário, os casos podem evoluir para óbito. Por conseguinte, o conhecimento da população local sobre as formas de tratamentos é imprescindível para o sucesso da cura da LV. A ausência de entendimento em relação à cura da LV é alarmante, particularmente em comunidades longe da extensão urbana ${ }^{25}$ como os bairros neste estudo.

Um estudo feito por Martins et al. ${ }^{27}$ mostra que em Buriticupu a doença se propaga durante todos os meses do ano, especialmente em abril, setembro, outubro e dezembro, onde a maior parte dos casos ocorreram na estação da estiagem. Isso é justificado pelo aumento da resistência dos vetores, assim sua incidência poderia ser influenciada por fatores geoclimáticos ${ }^{28}$.

Há diversos estudos no Maranhão que esclarecem sobre a correlação entre o clima e a umidade do ar. Numa análise, foi demonstrada que a temperatura acima de $20^{\circ} \mathrm{C}$ juntamente com alta umidade a $80 \%$ são oportunos para a expansão dos mosquitos transmissores de $\mathrm{LV}$, pois intensifica o progresso de decomposição de matéria orgânica gerando nutrientes para as larvas ${ }^{23}$. Em outro trabalho realizado por Monteiro et al. ${ }^{29}$ foi verificado que a periodicidade da LV ocorre em regiões mais quentes, onde o clima é seco.

Ações preventivas diante de doenças parasitárias necessitam de maior divulgação e estímulo por parte dos governantes e da própria sociedade, transformações atitudinais bem como o uso de hábitos profiláticos numa sociedade deve ser um objetivo a ser alcançado com o tempo ${ }^{13}$.

\section{CONCLUSÃO}

Constatou-se que, apesar de o município de Buriticupu apresentar características geoclimáticas favoráveis para a proliferação da LV, o baixo nível de instrução sobre a doença LV é um fator predominante entre os entrevistados. Tendo em vista que é fundamental para elevar o conhecimento da população em relação às doenças existentes na região, bem como o uso de medidas preventivas.

A educação em saúde constitui um dos pilares para a promoção da saúde em uma comunidade. A construção do saber científico com o auxílio de profissionais da área de saúde pode proporcionar a população seguir novos hábitos em seu cotidiano, além de torná-los agentes multiplicadores no controle e prevenção de doenças. Faz-se necessário, portanto, a otimização de ações preventivas junto ao poder público e serviços de saúde, com o comprometimento do repasse de informações efetivas para o controle da LV, principalmente no que diz respeito ao modo de transmissão, uso de medidas preventivas, divulgação e época de maior ocorrência.

\section{REFERÊNCIAS}

1. Cortes S, Vaz Y, Neves R, Maia C, Cardoso L, Campino L. Risk factors for canine leishmaniasis in an endemic Mediterranean region. Vet Parasitol. 2012;189(2-4):189-96.

2. Brito JA, Santos RA, Mendonça BC, Ribeiro RR. Avaliação do conhecimento sobre a leishmaniose visceral antes e depois de intervenção educacional em proprietários de cães da cidade de Cruz das Almas, Recôncavo da Bahia. Rev Ciênc Ext. 2015;11(2):104-14.

3. Brasil. Ministério da Saúde. Secretaria de Vigilância em Saúde. Departamento de Vigilância Epidemiológica. Manual de recomendações para diagnóstico, tratamento e acompanhamento de pacientes com co-infecção Leishmania-HIV. Brasília: Editora do Ministério da Saúde, 2011.

4. Brasil. Guia de orientação para vigilância de leishmaniose visceral canina (LVC). Santa Catarina. 2015.

5. Silva FS. Patologia e patogênese da leishmaniose visceral canina. $\mathrm{R}$ Trop $\mathrm{Ci}$ Agr Biol. 2007;1(1):20-31.

6. Bossler RS. Leishmaniose Visceral canina [monografia]. Porto Alegre: Faculdade de Veterinária - UFRS; 2012.

7. Brasil. Ministério da Saúde. Manual de vigilância da leishmaniose tegumentar. Brasília, 2010.

8. Santos LP, Nogueira MJ, Rezende CN, Ferreira RA. Doenças negligenciadas no município de Sabará: casos, portadores e percepções. Arq Cienc Saúde Unipar. 2017;21(3):155-62.

9. Marcondes M, Rossi CN. Leishmaniose visceral no Brasil. Braz J Vet Res Anim Sci. 2013;50(5):341-52.

10.Zuben APB, Donalisio MR. Dificuldades na execução das diretrizes do Programa de Vigilância e Controle da Leishmaniose Visceral em grandes municípios brasileiros. Cad Saúde Pública. 2016;32(6):e00087415.

11.Caldas AJM, Lisbôa LLC, Silva PF, Coutinha NPS, Silva TC. Perfil das crianças com leishmaniose visceral que evoluíram para óbito, falha terapêutica e recidiva em hospital de São Luís, Maranhão. Rev Pesq Saúde. 2013; 14(2):91-5.

12. Anversa L, Montanholi RJD Sabino DL. Avaliação do conhecimento da população sobre leishmaniose visceral. Rev Inst Adolfo Lutz. São Paulo, 2016; 75:1-8.

13. Borges BKA, Silva JA, Haddad JPA, Moreira EC, Magalhães DF, Ribeiro LML et al. Avaliação do nível de conhecimento e de atitudes preventivas da população sobre a leishmaniose visceral em Belo Horizonte, Minas Gerais, Brasil. Cad Saúde 
Pública. 2008;24(4):777-84.

14.IBGE. Censo Demográfico 2010, Área territorial brasileira. Rio de Janeiro: IBGE, 2017. Disponível em: https://cidades.ibge.gov.br/brasil/ma/ buriticupu. Acesso em: 16 de fevereiro de 2018.

15.Lima JS, Cajaíba RL, Martins JSC, Pereira KS, Sousa ES. Composição gravimétrica de resíduos sólidos em escolas públicas e privadas no município de Buriticupu, MA. Scientia Amazonia. 2017;6(3):11-6.

16. Moreira RCR, Rebêlo JMM, Gama MEA, Costa JML. Nível de conhecimento sobre leishmaniose tegumentar americana (LTA) e uso de terapias alternativas por populações de uma área endêmica da Amazônia do Maranhão, Brasil. Cad Saúd Pública. 2002;18(1):187-95.

17.Cavalcante IJM, Vale MR. Aspectos epidemiológicos da leishmaniose visceral (calazar) no Ceará no período de 2007 a 2011. Rev Bras Epidemiol. 2014;17(4):911-24.

18.Jayme MS, Wanderlei CL, Moura FFM, Castro JGD. Perfil epidemiológico dos casos de leishmaniose visceral em Palmas, Tocantins no período de 2007 - 2014. Rev Pat Tocantins. 2016;3(1):61-9.

19. Santos GM, Barreto MTS, Monteiro MJSD, Silva RVS, Jesus RLR, Silva HJN. Aspectos epidemiológicos e clínicos da leishmaniose visceral no estado do Piaú. Brasil. Rev Cien Desenvolv. 2017;10(2):142-53.

20.Lima AM, Alves LC, Faustino MA, Lira NMS. Percepção sobre o conhecimento e profilaxia das zoonoses e posse responsável em pais de alunos do pré-escolar de escolas situadas na comunidade localizada no bairro de Dois Irmãos na cidade do Recife (PE). Ciênc saúde coletiva. 2010; 15(Supl 1):1457-64.

21.Torres APC. Programa de orientação para controle de Leishmaniose Visceral canina [tese]. Jaboticabal: Faculdade de Ciências Agrárias e Veterinária - UNESP; 2017.

22. Souza VMMD, Hoffmann JL, Freitas MM, Brant JL, Araújo WN. Avaliação do conhecimento, atitudes e práticas sobre dengue no Município de Pedro Canário, Estado do Espírito Santo, Brasil, 2009: um perfil ainda atual. Rev Pan-Amaz Saúde. 2012;3(1):37-43.

23. Castro JM, Rodrigues SM, Tarso S, Costa FL, Rodrigues ACCP, Vieira LDF et al. Conhecimento, Percepções de Indivíduos em Relação à Leishmaniose Visceral Humana Como Novas Ferramentas de Controle. Ensaios Cienc Cienc Biol Agrar Saúde. 2016;20(2):93-103.

24.Genari ICC, Perri SHV, Pinheiro SR, Nunes CM. Atividades de educação em saúde sobre leishmaniose visceral para escolares. Vet e Zootec. 2012;19(1):99-107.
25.Gama Neto JL, Paulo CS, Passos MAB. Nível de conhecimentos sobre leishmaniose tegumentar americana entre moradores da vila do Apiaú, município de Mucajaí, Roraima, Brasil. Ambiente: Gestão e Desenvolvimento. 2012;4(1):59-68.

26. Sousa NA, Linhares CB, Pires FGB, Teixeira TC, Lima JS, Nascimento MLO. Perfil epidemiológico dos casos de leishmaniose visceral em Sobral-CE de 2011 a 2015. Sanare. 2018;17(1):51-7.

27. Martins LM. Ecoepidemiologia da leishmaniose tegumentar no Município de Buriticupu, Amazônia do Maranhão, Brasil, 1996 a 1998. Cad Saúde Pública;2004;20(3):735-43.

28. Silva AF, Latorre MRDO, Galati EAB. Fatores relacionados à ocorrência de leishmaniose tegumentar no Vale do Ribeira. Rev Soc Bras Med Trop. 2010;43(1):46-51.

29. Monteiro EM, Silva JCR, Costa RT, Costa DC, Barata RA, Paula EV et al. Leishmaniose visceral: estudo de flebotomíneos e infecção canina em Montes Claros, Minas Gerais. Rev Soc Bras Med Trop. 2005;38(2):147-52.

\section{CONFLITO DE INTERESSES}

Os autores declaram não haver conflitos de interesse.

\section{AUTOR PARA CORRESPONDENCIA}

\section{Gabriela de Sousa Lopes}

sousagaby347@gmail.com

Submetido em 17/09/2018

Aceito em 12/03/2019 